TAUP 2406-97

January 1997

\title{
The proton and the photon, who is probing who?
}

\author{
Aharon Levy \\ School of Physics and Astronomy \\ Raymond and Beverly Sackler Faculty of Exact Sciences \\ Tel-Aviv University, Tel-Aviv, Israel
}

\begin{abstract}
It is shown that by assuming Gribov factorization to hold at low $x$ one obtains a simple relation between the structure function of the proton and that of the photon. By interpreting an observed structure in deep inelastic scattering (DIS) of leptons on protons as belonging to the proton, one can relate it to the structure of the exchanged photon, and visa versa. Predictions are given for the structure function of real and virtual photons at low $x$ by using data on the proton structure function together with the Gribov factorization relations.
\end{abstract}




\section{Introduction}

Deep inelastic scattering (DIS) of leptons on protons are used as a tool to gain information about the structure of the proton. A neutral current (NC) reaction of the type $e p \rightarrow e X$ is interpreted naively as a process in which the electron radiates a virtual gauge boson, $\gamma$ or $Z^{0}$, which probes the structure of the proton. For low enough virtualities, expressed by the negative square of the four momentum transfer at the lepton vertex $Q^{2}$, one can neglect the $Z^{0}$ exchange. Thus we say that the virtual photon $\gamma^{*}$ is 'looking' at the proton and probing its structure. There is no question as to who 'looks' at who and thus if some structure is observed, it is attributed to the proton. The reason one does so is because one assumes that $\gamma^{*}$ has no structure. How well is this assumption justified?

Real photons are known to acquire structure when interacting with a proton. A photon fluctuates into a $q \bar{q}$ pair and as long as the fluctuation time $t_{f}$ is much larger than the interaction time $t_{i n t}$ the photon interacts with the proton through the $q \bar{q}$ pair [1]. A photon of energy $E_{\gamma}$ has a fluctuation time of:

$$
t_{f} \approx \frac{2 E_{\gamma}}{m_{q \bar{q}}^{2}}
$$

while the interaction time is determined by the radius $r_{p}$ of the proton: $t_{\text {int }} \sim r_{p}$. A virtual photon of virtuality $Q^{2}$ has a much shorter fluctuation time given by:

$$
t_{f} \approx \frac{2 E_{\gamma}}{m_{q \bar{q}}^{2}+Q^{2}}
$$

and thus does not have enough time to build up a structure before interacting with the proton.

However at small $x$ (the Bjorken scaling variable), the simple picture of DIS is complicated by the long chain of gluon and quark ladders which describes the process in Quantum Chromodynamics (QCD) [2]. In this long chain of partons along the ladder, where does one draw the line? Does one study the structure of the proton? of the photon? of both? Does it make at all sense to speak about the structure of virtual photons after the argument mentioned in the earlier paragraph where it was shown that the fluctuation time decreases with $Q^{2}$ ? That argument holds for the large $x$ region. At low $x$ the fluctuation time becomes approximately $Q^{2}$ independent:

$$
t_{f} \approx \frac{1}{2 m_{p} x}
$$

where $m_{p}$ is the proton mass and one assumes [3] $m_{q \bar{q}}^{2} \approx Q^{2}$. Thus for the low $-x$ region at HERA, one expects also highly virtual photons to acquire some structure before interacting with the proton. Thus we are back to the question of how to interpret the DIS measurements. Are we measuring the proton structure function $F_{2}^{p}$ or the photon structure function $F_{2}^{\gamma^{*}}$ ? Who is probing who?

It is clear that physics can not be frame dependent [4]. Thus it must be that both descriptions are correct and reflect the fact that cross sections are Lorenz invariant but time development is not [5]. This means that it shouldn't matter whether one interprets the cross section measurements as yielding the proton or the photon structure function. By extracting 
one of them from the cross section measurement, there should be a relation allowing to obtain the other. On the other hand we know that at least as far as a real photon is concerned, its structure function behaves very differently from that of the proton one. As an example one can mention the $Q^{2}$ scaling violation which is positive in the photon case for all values of $x$, while for the proton they change from positive to negative scaling violations as one moves to higher $x$ values [6].

The purpose of this note is to suggest a way of relating the proton and the photon structure function in the low $-x$ region, where the interpretation of the results can be ambiguous. By extending the Gribov factorization [0] from the real photon to the virtual photon case one can obtain $F_{2}^{\gamma}$ and $F_{2}^{\gamma^{*}}$ from the measured $F_{2}^{p}$ in the low- $x$ region.

\section{Gribov factorization}

Gribov factorization is based on the assumption that at high energies the total cross section of two interacting particles is determined by the property of the universal pomeron trajectory. This implies relations between total cross section of various particles. Gribov factorization can be used [7, 8] to relate the total $\gamma \gamma$ cross section, $\sigma_{\gamma \gamma}$, with that of photoproduction, $\sigma_{\gamma p}$, and that of $p p, \sigma_{p p}$, all at the same center of mass energy squared $W^{2}$ :

$$
\sigma_{\gamma \gamma}\left(W^{2}\right)=\frac{\sigma_{\gamma p}^{2}\left(W^{2}\right)}{\sigma_{p p}\left(W^{2}\right)} .
$$

This relation is approximately borne out [9] with the available measurements of $\sigma_{\gamma \gamma}$.

In case one of the photons is virtual, and assuming that Gribov factorization is applicable also for virtual photons, one can write:

$$
\sigma_{\gamma^{*} \gamma}\left(W^{2}, Q^{2}\right)=\frac{\sigma_{\gamma^{*} p}\left(W^{2}, Q^{2}\right) \cdot \sigma_{\gamma p}\left(W^{2}\right)}{\sigma_{p p}\left(W^{2}\right)} .
$$

The interest in the low- $x$ region stems from the fact that low $x$ for a given $Q^{2}$ value means high center of mass energy $W$, since they are related through:

$$
W^{2}=Q^{2}\left(\frac{1}{x}-1\right)-m_{p}^{2} \simeq \frac{Q^{2}}{x} .
$$

In this region one can connect the proton structure function $F_{2}^{p}$ with the total $\gamma^{*} p$ cross section:

$$
\begin{aligned}
\sigma_{\gamma^{*} p}\left(W^{2}, Q^{2}\right) & =\frac{4 \pi^{2} \alpha}{Q^{2}} \frac{1}{1-x}\left(1+\frac{4 m_{p}^{2} x^{2}}{Q^{2}}\right) F_{2}^{p}\left(x, Q^{2}\right) \\
& \approx \frac{4 \pi^{2} \alpha}{Q^{2}} F_{2}^{p}\left(x, Q^{2}\right)
\end{aligned}
$$

Similarly one can relate the photon structure function $F_{2}^{\gamma}$ to the total $\gamma^{*} \gamma$ cross section:

$$
\sigma_{\gamma^{*} \gamma}\left(W^{2}, Q^{2}\right) \approx \frac{4 \pi^{2} \alpha}{Q^{2}} F_{2}^{\gamma}\left(x, Q^{2}\right)
$$


The approximate signs are well justified for the low- $x$ region. Using equation (5) one gets:

$$
F_{2}^{\gamma}\left(x, Q^{2}\right)=F_{2}^{p}\left(x, Q^{2}\right) \frac{\sigma_{\gamma p}\left(W^{2}\right)}{\sigma_{p p}\left(W^{2}\right)} .
$$

This last equation connects the proton and the real photon structure function at low $x$. By measuring one of them, the other can be determined through relation (10). Please note at this point that $x$ should be treated as a measure of the center of mass energy available in the interaction, as given in equation (6).

The next step is to extend relation (5) to the case where both photons are virtual, one with virtuality of $Q^{2}$ and the other with $P^{2}$. Assuming Gribov factorization to hold also for this case, one can write:

$$
\sigma_{\gamma^{*} \gamma^{*}}\left(W^{2}, Q^{2}, P^{2}\right)=\frac{\sigma_{\gamma^{*} p}\left(W^{2}, Q^{2}\right) \cdot \sigma_{\gamma^{*} p}\left(W^{2}, P^{2}\right)}{\sigma_{p p}\left(W^{2}\right)} .
$$

Just like above, we can replace some of the cross sections with structure functions to obtain:

$$
F_{2}^{\gamma^{*}}\left(x, Q^{2}, P^{2}\right)=F_{2}^{p}\left(x, Q^{2}\right) \frac{\sigma_{\gamma^{*} p}\left(W^{2}, P^{2}\right)}{\sigma_{p p}\left(W^{2}\right)} .
$$

This can also be expressed as:

$$
F_{2}^{\gamma^{*}}\left(x, Q^{2}, P^{2}\right)=\left(\frac{4 \pi^{2} \alpha}{P^{2}}\right) \frac{F_{2}^{p}\left(x, Q^{2}\right) \cdot F_{2}^{p}\left(x, P^{2}\right)}{\sigma_{p p}\left(W^{2}\right)} .
$$

Once again we obtained at low $x$ a relation between the measured proton structure function and that of the virtual photon. Thus equations (10) and (13) suggest that indeed at low $x$ both interpretation of the DIS cross sections as describing the structure of the proton or that of the photon are correct.

\section{$3 \quad$ Expected $W$ dependence of cross sections}

\subsection{The total $\gamma \gamma$ cross section, $\sigma_{\gamma \gamma}\left(W^{2}\right)$}

Donnachie and Landshoff (DL) [10] have shown that all data on hadron hadron cross sections can be expressed in the Regge picture as a sum of two terms coming from contributions of the pomeron and the reggeon trajectories. The contribution of the pomeron term is proportional to $\left(W^{2}\right)^{\Delta}$ where the pomeron intercept is expressed as $\alpha_{P}(0)=1+\Delta$. By fitting all available total cross section data, they obtained the value $\Delta=0.08$. The measurements at HERA of the total photoproduction cross section [11] showed that this value of $\Delta$ can explain also $\gamma p$ cross sections. Assuming that the pomeron term dominates, $\sigma_{\gamma p}\left(W^{2}\right)=0.068\left(W^{2}\right)^{0.08}$ and $\sigma_{p p}\left(W^{2}\right)=21.7\left(W^{2}\right)^{0.08}$, where both coefficients are such that the resulting cross section is in milibarns and $W$ is in $\mathrm{GeV}$. By using relation (4) one obtains [12]:

$$
\sigma_{\gamma \gamma}\left(W^{2}\right)=0.21 \times 10^{-3}\left(W^{2}\right)^{0.08},
$$

where again the cross section is expressed in milibarns. This expression thus indicates that the energy behaviour of the total $\gamma \gamma$ cross sections is the same as any other hadron hadron or photoproduction cross section behaviour. 


\subsection{The total $\gamma^{*} \gamma$ cross section, $\sigma_{\gamma^{*} \gamma}\left(W^{2}, Q^{2}\right)$}

Equation (5) can be used to get the relation:

$$
\sigma_{\gamma^{*} \gamma}\left(W^{2}, Q^{2}\right)=3.1 \times 10^{-3} \sigma_{\gamma^{*} p}\left(W^{2}, Q^{2}\right),
$$

which predicts that the $\gamma^{*} \gamma$ total cross sections behaves at large energies in the same way as the $\gamma^{*} p$ cross section. The HERA data [13] can be well described by $\sigma_{\gamma^{*} p}\left(W^{2}, Q^{2}\right) \sim\left(W^{2}\right)^{\Delta\left(Q^{2}\right)}$ [14], and thus one expects also:

$$
\sigma_{\gamma^{*} \gamma}\left(W^{2}, Q^{2}\right) \sim\left(W^{2}\right)^{\Delta\left(Q^{2}\right)} .
$$

Since $\Delta\left(Q^{2}\right)$ increases with $Q^{2}$ from the value of 0.08 at $Q^{2} \sim 0$ to a value of about 0.3 at $Q^{2} \sim$ $20 \mathrm{GeV}^{2}$, we expect that also the $\gamma^{*} \gamma$ total cross section will have a steeper $W$ behaviour as $Q^{2}$ increases.

\subsection{The total $\gamma^{*} \gamma^{*}$ cross section, $\sigma_{\gamma^{*} \gamma^{*}}\left(W^{2}, Q^{2}, P^{2}\right)$}

In the case where both photons are virtual the energy behaviour will depend on both $Q^{2}$ and $P^{2}$. Using equation (11), one gets:

$$
\sigma_{\gamma^{*} \gamma^{*}}\left(W^{2}, Q^{2}, P^{2}\right) \sim\left(W^{2}\right)^{\Delta\left(Q^{2}\right)+\Delta\left(P^{2}\right)-\Delta(0)} .
$$

Since $\Delta\left(P^{2}\right)>\Delta(0)$, this means that the $\gamma^{*} \gamma^{*}$ cross section is expected to have a steeper $W$ dependence than the total $\gamma^{*} p$ one.

\section{Comparison with existing data}

We compare the Gribov factorization relations to data from two experiments which performed a double tag measurement and obtained $\sigma_{\gamma^{*} \gamma^{*}}\left(W^{2}, Q^{2}, P^{2}\right)$. One is the TPC collaboration [15] which tagged both photons with a virtuality of $Q^{2}=P^{2}=0.3 \mathrm{GeV}^{2}$. The other is the PLUTO collaboration [16] which tagged one photon at $Q^{2}=5 \mathrm{GeV}^{2}$ and the other at $P^{2}=0.35 \mathrm{GeV}^{2}$.

Figure 1 presents the total cross section data as function of $W$. In order to calculate the expectations coming from the Gribov factorization relation (11) we use the DL parameterization of the $p p$ total cross section. For the $\gamma^{*} p$ cross section we use the ALLM [17] parameterization which gives a good description of all the measured data in the $Q^{2}$ range between 0.3-2000 $\mathrm{GeV}^{2}$. The curves appearing in the figure are obtained using the above parameterizations. Since the relations from the Gribov factorization are expected to be valid in the low $-x$ region, the value of $W$ at which it can be applied is $Q^{2}$ dependent. If we call 'low- $x$ ' as $x \sim 10^{-2}$, we expect the factorization to work for $W \sim 5 \mathrm{GeV}$ for $Q^{2} \sim 0.3 \mathrm{GeV}^{2}$ and $W \sim 22 \mathrm{GeV}$ for $Q^{2} \sim 5 \mathrm{GeV}^{2}$. As can be seen from the figure, the TPC data are in the range of validity of the Gribov factorization and good agreement between the expectations and the data is obtained. The PLUTO data are at lower $W$ values, where one can still see a drop with energy toward the place where it would start rising again. 

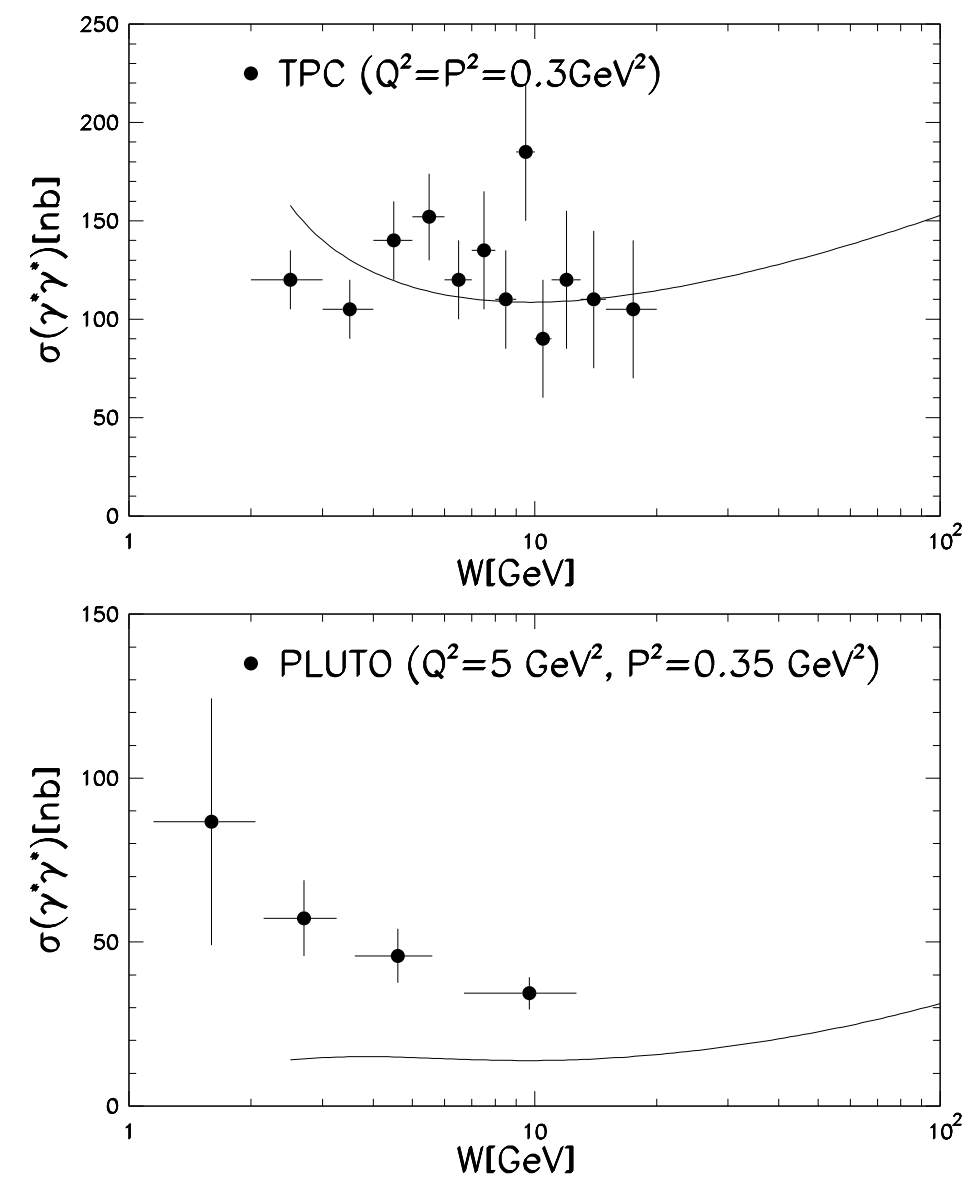

Figure 1: $\quad$ The total $\gamma^{*} \gamma^{*}$ cross section as function of $W$ for the TPC data $\left(Q^{2}=P^{2}=0.3\right.$ $\mathrm{GeV}^{2}$ ) (upper plot) and for the PLUTO data $\left(Q^{2}=5 \mathrm{GeV}^{2}, P^{2}=0.35 \mathrm{GeV}^{2}\right)$ (lower plot). The curves are the expectations from the Gribov factorization relation.

\section{The photon structure function}

\subsection{Real photon structure function, $F_{2}^{\gamma}$}

The structure function of a real photon was measured in a $Q^{2}$ range of $0.24-390 \mathrm{GeV}^{2}$. Most measurements, except those in the low $Q^{2}$ region, are in the relatively high $-x$ region. The data are plotted in figure 2 as function of $x$ for fixed $Q^{2}$ values. In general, the structure function values seem to be decreasing as $x$ gets smaller. There is a clear lack of data in the region $x<0.1$. The lines in the figure are the result of using the Gribov factorization relation (10). Instead of using the available data on $F_{2}^{p}, \sigma_{\gamma p}, \sigma_{p p}$ and extrapolate/interpolate the values to the kinematic region where the $\gamma \gamma$ data is measured, we used the DL parameterization for the $\gamma p$ and $p p$ cross sections and the ALLM parameterization for $F_{2}^{p}$. With the DL parameterization one obtains a simple relation:

$$
F_{2}^{\gamma} / \alpha=0.43 F_{2}^{p}
$$




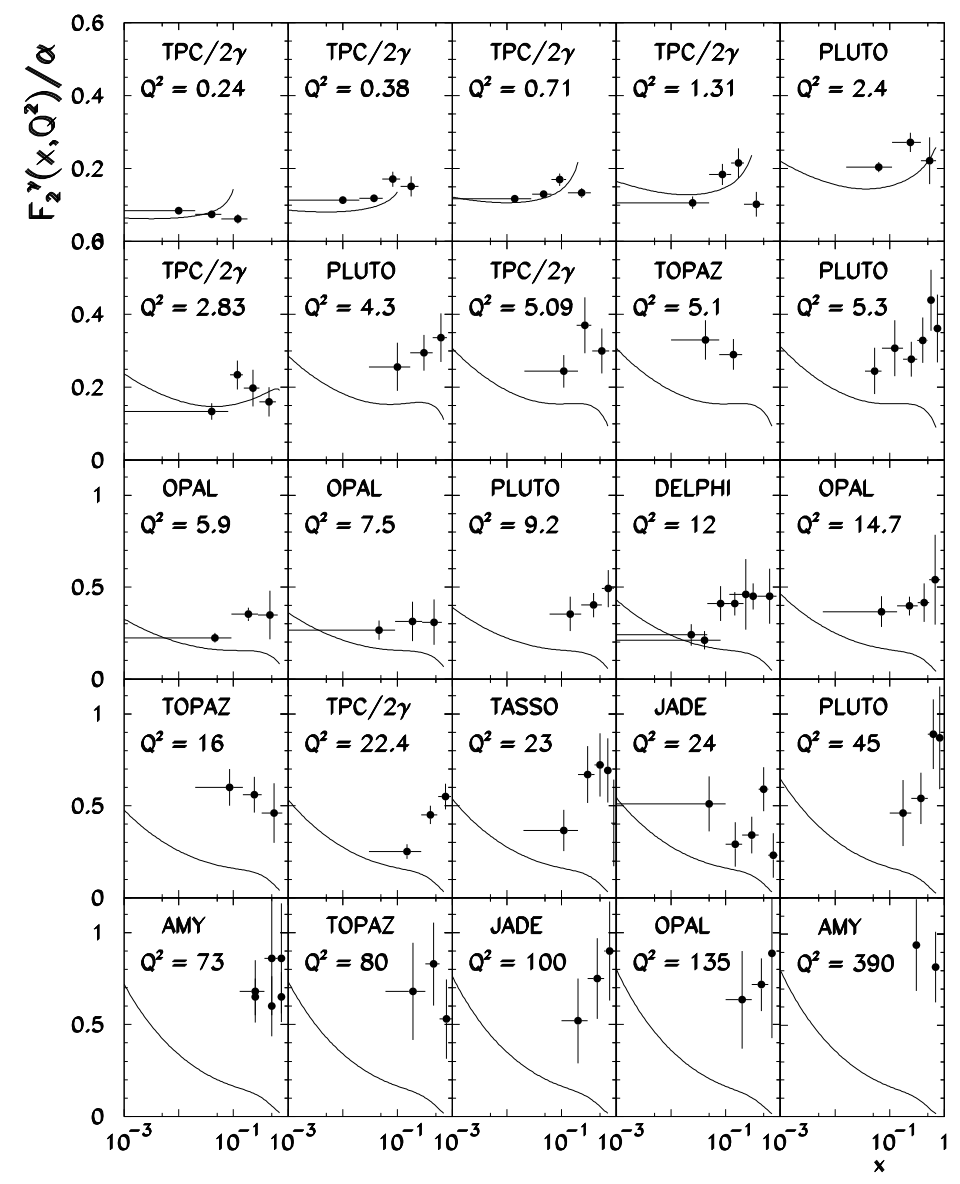

Figure 2: A compilation of the data on the real photon structure function, $F_{2}^{\gamma} / \alpha$, as a function of $x$ for fixed $Q^{2}$ values. The curves are the expectations from the Gribov factorization relation.

In the low $Q^{2}$ region, where low- $x$ data are available for the photon structure function, the lines agree with the data. At higher $Q^{2}$ regions, the predictions at high $x$ fall below the data as expected since in the large $-x$ region factorization is not expected to hold. In the low $-x$ region, the shape of $F_{2}^{\gamma}$ should follow exactly that of $F_{2}^{p}$ according to equation (18). The parameterization of the photon parton distributions given in [18] has this feature in the low- $x$ region. It would be most desirable to get data on the photon structure function in the low $-x$ region to confirm this relation.

\subsection{Virtual photon structure function, $F_{2}^{\gamma^{*}}$}

There exist no measurement of $F_{2}^{\gamma^{*}}$ in the low $-x$ region. We will use relation (12) to calculate the predictions of the Gribov factorization for some specific $Q^{2}$ and $P^{2}$ values, shown in figure 3 . One sees a steep rise of the structure function with decreasing $x$ which is steeper than that observed for the proton structure function. It is also interesting to note that even for a virtual photon of $P^{2}=10 \mathrm{GeV}^{2}$, its structure function has a relatively high value at low enough $x$ provided the 'probing' photon is highly virtual compared to the 'probed' photon. 


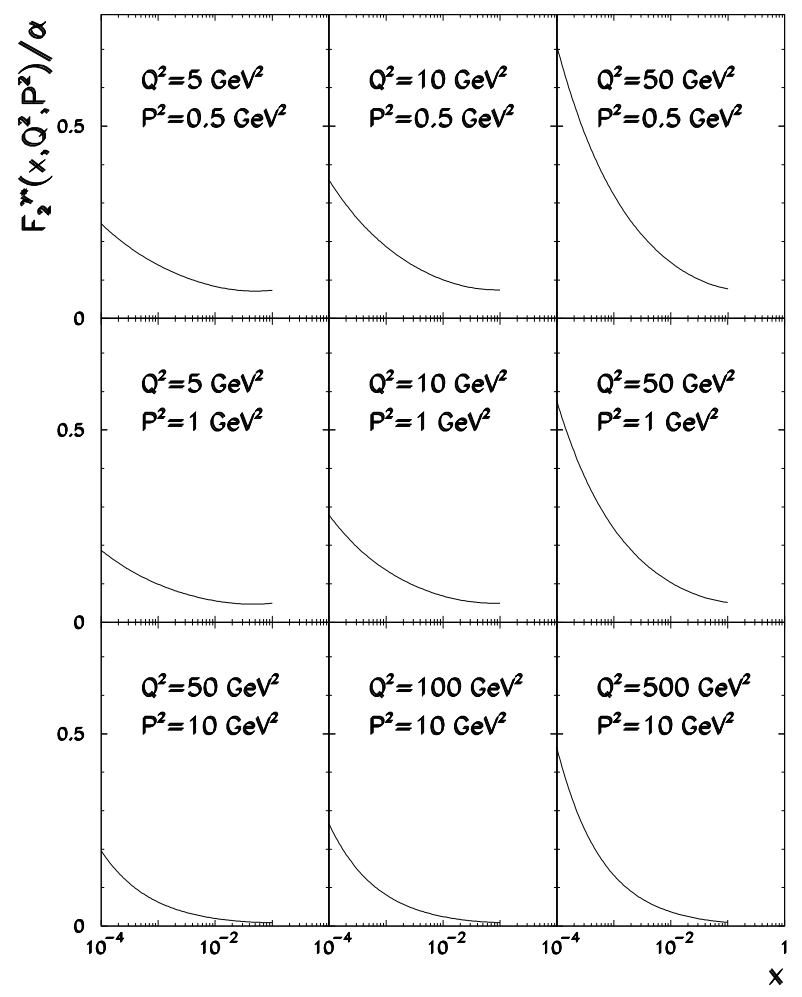

Figure 3: The predicted behaviour of the virtual photon structure function as function of $x$, using the Gribov factorization relation, for values of $Q^{2}$ and $P^{2}$ as indicated in the figure.

\section{Discussion and conclusions}

Gribov factorization has been applied for two cases where data exist and in the region of its expected validity the results are consistent with the data. One can use the measurements of $F_{2}^{p}$ at low $x$ in order to predict $F_{2}^{\gamma}$ in that $x$ region. Predictions have been also given for the structure function of virtual photons for some values of $P^{2}$.

The title of the paper indicated a problem of how to interpret at low $x$ the results of the DIS experiments as to who is being probed by who. Since in DIS one measures cross sections, in principle this question should not matter since the result cannot be frame dependent. This however means that by interpreting the result as coming from the structure of the proton, one should be able to learn about the structure of the photon. We showed here that if one can assume Gribov factorization to hold also for the case of a virtual photon, one can obtain a simple relation between the proton and the photon structure function. Thus by measuring one of them, the other is determined through the factorization relation.

The simple relation between the photon and proton structure function obtained here relies on the extension of Gribov factorization to the case of a virtual photon. This implies a factorizable pomeron also in DIS processes, which so far seems to be borne out experimentally at HERA [19]. Experimental verification of the relations between the photon and proton structure functions given above would be another support for the factorizable pomeron in DIS. However it should 
be noted that factorization breaking of the pomeron in DIS would mean that the relations between the proton and photon structure function are not as simple as those obtained in this paper.

We can conclude that at large $x$ the photon is probing the proton. At low $x$ they seem to talk to each other: probing one teaches us about the other.

\section{Acknowledgments}

It is a pleasure to acknowledge fruitful discussions with H. Abramowicz, J. D. Bjorken, L. L. Frankfurt, E. G. Gurvich and M. Krawczyk.

This work was partially supported by the German-Israel Foundation (GIF).

\section{References}

[1] B.L. Ioffe, Phys. Lett. 30 (1969) 123.

[2] R. G. Roberts, The structure of the proton, Cambridge University Press, 1990.

[3] H. Abramowicz, L. Frankfurt, M. Strikman, Interplay of hard and soft physics in small $x$ DIS, DESY 95-045 (1995). Published in SLAC Summer Inst. (1994) 539.

[4] J.D Bjorken, Proceedings of the International Workshop on DIS and related subjects, Eilat, 1994, p. 151.

[5] L. Frankfurt, private communication.

[6] See e.g. H. Abramowicz, Rapporteur talk in Proceedings of the XXVIII International Conference on High Energy Physics, Warsaw, Poland, 1996.

[7] V. N. Gribov, L. Ya. Pomeranchuk, Phys. Rev. Lett. 8 (1962) 343.

[8] S. J. Brodsky, T. Kinoshita, H. Terazawa, Phys. Rev. D4 (1971) 1532;

J. L. Rosner, in ISABELLE Physics Prospects, BNL Report 17522(1972) 316.

[9] See e.g. Ch. Berger, W. Wagner, Phys. Rep. C146 (1987) 1.

[10] A. Donnachie, P. V. Landshoff, Phys. Lett. B296 (1992) 227.

[11] ZEUS Collab., M. Derrick et al., Phys. Lett. B293 (1992) 465;

H1 Collab., T. Ahmed et al., Phys. Lett. B299 (1992) 374.

[12] See also G. A. Schuler, T. Sjöstrand, A scenario for high-energy $\gamma \gamma$ interactions, CERNTH/96-119 (1996). 
[13] H1 Collab., S. Aid et al., Nucl. Phys. B470 (1996) 3;

ZEUS Collab., M. Derrick et al., Zeit. Phys. C72 (1996) 399.

[14] See e.g. A. Levy, Rapporteur talk in Proceedings of the International Europhysics Conference on High Energy Physics, Brussels, Belgium 1995, p. 699.

[15] TPC Collab., D. Bintinger et al., Phys. Rev. Lett. 54 (1985) 763.

[16] PLUTO Collab., Ch. Berger et al., Phys. Lett. B142 (1984) 119.

[17] H. Abramowicz et al., Phys. Lett. B269 (1991) 465;

A. Marcus, Energy dependence of the $\gamma^{*} p$ cross section, M.Sc. Thesis, Tel-Aviv University, TAUP 2350-96 (1996).

[18] G. A. Schuler, T. Sjöstrand, Zeit. Phys. C68 (1995) 607.

[19] H1 Collab., T. Ahmed et al., Phys. Lett. B348 (1995) 681;

ZEUS Collab., M. Derrick et al., Zeit. Phys. C68 (1995) 569. 